\title{
Chest wall thickness and depth to vital structures in paediatric patients - implications for prehospital needle decompression of tension pneumothorax
}

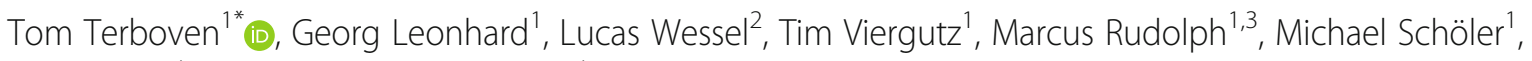
Meike Weis ${ }^{4}$ and Holger Haubenreisser ${ }^{4}$

\begin{abstract}
Background: Recommendations regarding decompression of tension pneumothorax in small children are scarce and mainly transferred from the adult literature without existing evidence for the paediatric population. This CTbased study evaluates chest wall thickness, width of the intercostal space (ICS) and risk of injury to vital structures by needle decompression in children.

Methods: Chest wall thickness, width of the intercostal space and depth to vital structures were measured and evaluated at 2nd ICS midclavicular (MCL) line and 4th ICS anterior axillary line (AAL) on both sides of the thorax using computed tomography (CT) in 139 children in three different age groups $(0,5,10$ years).

Results: Width of the intercostal space was significantly smaller at the 4th ICS compared to the 2nd ICS in all age groups on both sides of the thorax. Chest wall thickness was marginally smaller at the 4th ICS compared to the 2nd ICS in infants and significantly smaller at 4th ICS in children aged 5 years and 10 years. Depth to vital structure for correct angle of needle entry was smaller at the 4th ICS in all age groups on both sides of the thorax. Incorrect angle of needle entry however is accompanied by a higher risk of injury at 2nd ICS. Furthermore, in some children aged 0 and 5 years, the heart or the thymus gland were found directly adjacent to the thoracic wall at 2nd ICS midclavicular line.

Conclusion: Especially in small children risk of iatrogenic injury to vital structures by needle decompression is considerably high. The 4th ICS AAL offers a smaller chest wall thickness, but the width of the ICS is smaller and the risk of injury to the intercostal vessels and nerve is greater. Deviations from correct angle of entry however are accompanied by higher risk of injury to intrathoracic structures at the 2nd ICS. Furthermore, we found the heart and the thymus gland to be directly adjacent to the thoracic wall at the 2nd ICS MCL in a few children. From our point of view this puncture site can therefore not be recommended for decompression in small children. We therefore recommend 4 th ICS AAL as the primary site of choice.
\end{abstract}

Keywords: Tension pneumothorax, Needle decompression, Children, Paediatric, Chest wall thickness, Complications

\footnotetext{
* Correspondence: tom.terboven@umm.de

'Department of Anaesthesiology and Intensive Care Medicine, University

Medical Center Mannheim, Theodor-Kutzer-Ufer 1-3, 68167 Mannheim,

Germany

Full list of author information is available at the end of the article
}

(c) The Author(s). 2019 Open Access This article is distributed under the terms of the Creative Commons Attribution 4.0 International License (http://creativecommons.org/licenses/by/4.0/), which permits unrestricted use, distribution, and reproduction in any medium, provided you give appropriate credit to the original author(s) and the source, provide a link to the Creative Commons license, and indicate if changes were made. The Creative Commons Public Domain Dedication waiver (http://creativecommons.org/publicdomain/zero/1.0/) applies to the data made available in this article, unless otherwise stated. 


\section{Background}

Traumatic or spontaneous tension pneumothorax is a potentially fatal event that requires immediate decompression. Currently recommended interventions for decompression are either needle thoracostomy or open finger thoracostomy $[1,2]$. Needle thoracostomy is generally easier to learn, faster to perform and less invasive than surgical decompression. It therefore represents the preferred first line technique for many emergency providers and is recommended in several trauma guidelines $[2,3]$. However, recent ATLS 10th edition guidelines suggest 4th/5th ICS mid-axillary line as preferable to 2 nd ICS in adults and recommends same management for pneumothorax in children, except for needle or tube size. 2nd ICS MCL is still recommended as a possible insertion site in children [4]. There is no reference in the APLS 6th edition on depth of insertion and it recommends 2nd ICS insertion site only [5]. Commonly recommended puncture techniques are insertion sagittal to the chest wall at the 2nd intercostal space (ICS) in the midclavicular line (MCL) and perpendicular to the chest wall at the 4th or 5th ICS anterior axillary line (AAL) or midaxillary line (MAL). However, failed decompression is a commonly reported phenomenon in needle thoracostomy [6]. Therefore, in recent years, several studies examining chest wall thickness (CWT) at the recommended insertion sites have been conducted in adult patients and found commonly used cannulas being too short for successful decompression in a high proportion of patients [6]. This has led to the recommendation of using longer $7-8 \mathrm{~cm}$ catheters for needle thoracostomy in adult patients [7-9]. Even though this increases the likelihood of successful decompression, it also increases the risk of injuring underlying vital structures like large intrathoracic vessels or the heart because of the possibility of deeper insertion [9]. Due to the smaller anatomic structures, tension pneumothorax represents a particular challenge in paediatric patients. Open finger thoracostomy in the very narrow intercostal spaces in children requires smaller instruments and some surgical skills, which are often not available in the prehospital setting. Especially in small children it is accompanied by the risk of too large incisions with leakage of air along chest tubes. Furthermore, whilst the technique is similar to an adult, invasive paediatric critical procedures are often associated with cognitive hurdles and dissonance. Little is known about the required insertion depth of a needle for decompression in paediatrics or the likelihood of injuring underlying vital structures. Because of the narrow intercostal space, the risk of injury to the intercostal vessels and nerves has to be taken into consideration when performing needle decompression. We aimed to evaluate the required depth for successful decompression, defined as the distance from skin to pleural space, whilst minimizing iatrogenic underlying structure injury.
Therefore, the primary aim of this study was evaluation of the insertion site recommended by APLS guidelines (2nd ICS MCL) regarding risk of injury to intrathoracic vital structures. Secondary aims were assessing required insertion depth of the needle for successful decompression and measuring width of the intercostal space to study if finger thoracostomy is possible. Furthermore, we investigated the same questions at the 4th ICS AAL as an alternative insertion site.

\section{Methods}

Inclusion criteria were meeting one of the required age groups and availability of a thoracic CT scan in the local picture communication and archiving system. A total of 197 paediatric patients referred for thoracic CT with various indications were initially included in this study. We excluded all patients with a condition that made one or more of the measurements impossible or inaccurate. Consequently 58 patients were excluded due to various pulmonary pathologies which made the required measurements impossible or invalid. Most of the excluded patients were infants with large intrathoracic pathologies or conditions resulting in mediastinal shift. Details on reasons for exclusion are shown in Table 1. The remaining 139 patients were included in three study groups aged 0,5 and 10 years.

\section{Data acquisition}

All patients had paediatric thoracic CT protocol (2nd generation DSCT, Siemens Somatom Definition Flash, Siemens Healthineers, Forchheim, Germany) or 16 slice MSCT (Siemens Emotion 16, Siemens Healthineers, Forchheim, Germany). All examinations were reconstructed with $1.5 \mathrm{~mm}$ slice thickness, increments of $1.0 \mathrm{~mm}$, a dedicated lung reconstruction kernel (I70s (DSCT) or B70s (MSCT)) and soft tissue reconstruction kernel (I30s (DSCT) or B30s (MSCT)). The reconstruction kernels on the DSCT system utilized an iterative reconstruction algorithm with a strength level of 2 . Image data were imported into a PACS Workstation (Aycan OsiriX PRO v.2.10, Aycan Digitalsysteme GmbH, Würzburg, Germany) and evaluated in an axial plane, as well as using multiplanar reconstructions (MPR). We used the orthogonal MPR feature in our DICOM viewer (Osirix). This allows for accurate measurements in all planes, as the data are built from $1.5 \mathrm{~mm}$ slices with 1.0 $\mathrm{mm}$ increment. The overlapping datasets ensure that the MPR images are true to the original, with no drawbacks compared to axial/coronal/sagittal reconstructions from the scanner. All CTs were reviewed and measurements recorded by one specialist in paediatric radiology.

\section{Measurements}

Measurements were made at 2nd ICS MCL and 4th ICS AAL on both sides of the thorax. ICS width was 
Table 1 Reasons for exclusion

\begin{tabular}{|c|c|c|c|c|}
\hline Reason for exclusion & $\mathrm{n}$ (0 years) & $\mathrm{n}$ (5 years) & $\mathrm{n}$ (10 years) & $\mathrm{n}$ (total) \\
\hline Mediastinal shift & 12 & 1 & 1 & 14 \\
\hline Pulmonary infiltration & 11 & 1 & 0 & 12 \\
\hline CPAM & 8 & 0 & 0 & 8 \\
\hline Pulmonary bullae & 6 & 0 & 0 & 6 \\
\hline Pleural effusion/empyema & 3 & 2 & 0 & 5 \\
\hline Poor image quality & 1 & 3 & 0 & 4 \\
\hline Spinal misalignment & 1 & 1 & 1 & 3 \\
\hline Emphysema & 3 & 0 & 0 & 3 \\
\hline Congenital diaphragmatic hernia & 2 & 0 & 0 & 2 \\
\hline Intrathoracic mass & 1 & 0 & 0 & 1 \\
\hline Total & 48 & 8 & 2 & 58 \\
\hline
\end{tabular}

measured from the inferior border of the superior rib to the superior edge of the inferior rib. Chest wall thickness and the depth to the closest vital structure were measured in various directions: in the sagittal plane at the 2nd ICS MCL $\left(\mathrm{MCL}_{\mathrm{sag}}\right)$, perpendicular to the chest wall at the 2nd and 4th ICS (MCL perp, AAL perp) and in a linear direction to the closest vital structure at the 2nd

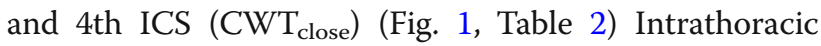
structures classified as "vital structures" are shown in Table 3. Lung parenchyma was not classified as a vital structure. Since a pneumothorax in the prehospital setting can hardly be confirmed with a $100 \%$ accuracy and an accidental puncture of the lung parenchyma is possible, we chose to define the intraparenchymal lung vessels up to the segmental vessels as vital structures. Chest wall thickness was measured from the skin surface to the pleural cavity. Depth to vital structure was defined as the distance from the skin surface to the intersection with a vital structure. In an additional measurement, the closest vital structure to skin surface at the insertion sites was identified visually. The distance from skin surface to this reference point at the corresponding puncture site was measured. The distance for the worst case scenario; an insertion leading directly to the closest vital structure, was determined $\left(\mathrm{DVS}_{\text {close }}\right)$. By subtracting CWT from DVS, the so called "intrapleural safety zone (ISZ)" was calculated. ISZ represents the intrathoracic distance from entering the pleural space up to the beginning of the closest vital structure (Fig. 1).

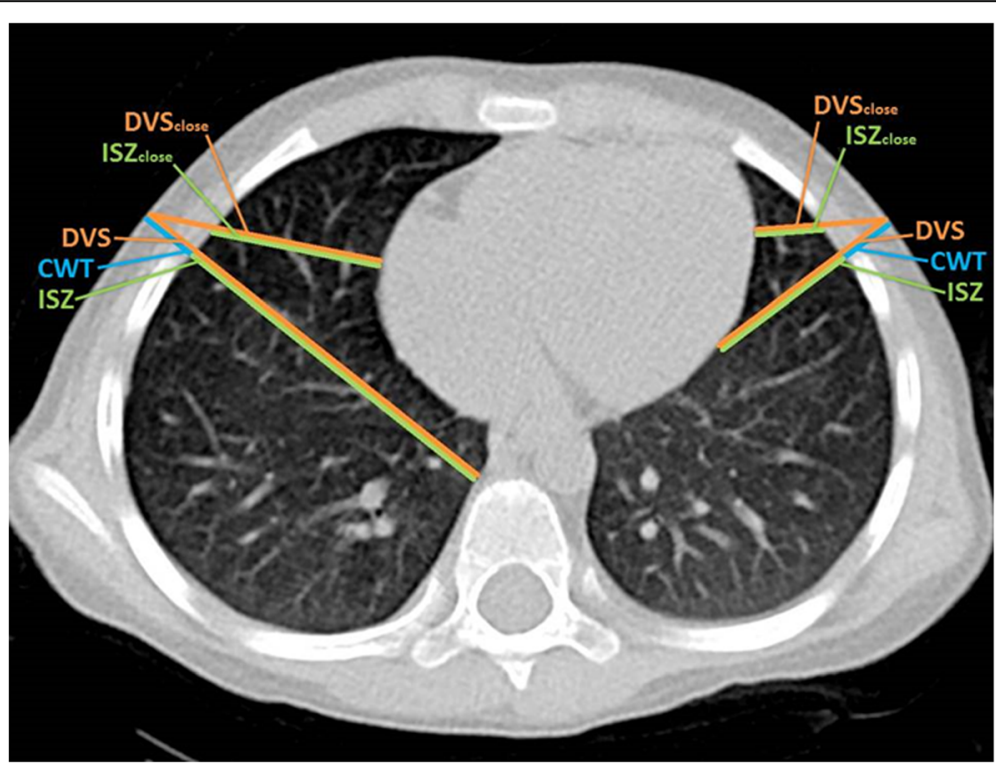

Fig. 1 Graphical display of measurements taken. 4th ICS AAL. DVS: depth to vital structure, CWT: chest wall thickness, ISZ: intrapleural safety zone. Subscript "close" indicating measurements for a misguided puncture directed at the closest vital structure 
Table 2 Description of measurements

\begin{tabular}{|c|c|}
\hline Measurement & Description \\
\hline Width of the intercostal space & From the inferior border of the superior rib to the superior edge of the inferior rib \\
\hline Chest Wall Thickness (CWT) & Skin to pleural space \\
\hline Depth to vital structure (DVS) & $\begin{array}{l}\text { Skin to the intersection of the insertion line (see "directions of insertion") with an intrathoracic } \\
\text { vital structure (see Table 3) }\end{array}$ \\
\hline Intrapleural Safety Zone (ISZ) & $\begin{array}{l}\text { Pleural space to the intersection with an intrathoracic vital structure (DVS - CWT), representing } \\
\text { the intrathoracic distance to a vital structure }\end{array}$ \\
\hline \multicolumn{2}{|l|}{ Directions of insertion } \\
\hline Sagittal & Insertion in the sagittal plane \\
\hline Perpendicular & Insertion perpendicular to the chest wall \\
\hline Close & $\begin{array}{l}\text { The closest vital structure to the point of insertion was identified visually. DVS was then measured } \\
\text { from this point of reference to skin surface. }\end{array}$ \\
\hline
\end{tabular}

\section{Statistical analysis}

Statistical analysis was performed using JMP 13.0 (SAS Institute Inc., Cary, NC, USA). Normally distributed data were identified using the Shapiro-Wilk W test. Continuous variables are presented as mean \pm standard deviation and median and interquartile range. Comparison was made using the Mann-Whitney $U$ test. $P$-values $<0.05$ were considered statistically significant.

\section{Results}

\section{Demographics}

139 patients were included in the final analysis. 50 Children aged 0 years, 47 children aged 5 years and 42 children aged 10 years. Mean ages in the three groups were $0.42( \pm 0.32)$ years, $5.48( \pm 0.28)$ years and $10.45( \pm 0.30)$ years. Demographic data are shown in Table 4.

\section{0-year-old children}

The width of the ICS was significantly larger at 2nd ICS compared to 4th ICS (right: $p<0.05$, left: $p<0.05$ ). Chest wall thickness was slightly but not significantly greater at 2nd ICS than at 4th ICS. Mean required depth of puncture for successful decompression was approximately $1.4-1.6 \mathrm{~cm}$ at all puncture sites. DVS was significantly greater at 2nd ICS on both sides of the thorax (right: $p<0.05$, left: $p<0.05$ ), $\mathrm{DVS}_{\text {close }}$ however (a misguided puncture directly at the closest vital structure) was significantly smaller at 2 nd ICS (right: $p<0.05$, left: $p<0.05$ ). The safe zone from penetration of the pleura to the intersection with the next vital structure (ISZ) was significantly

Table 3 Intrathoracic structures defined as "vital structures"

Pericardium
Aorta
SVC, IVC
Pulmonary vessels including larger intraparenchymal branches (with the
smallest easily visualized on CT-scan being the segment arteries)
Thymus gland

greater for correct angles of puncture (sagittal at 2nd ICS MCL and perpendicular at 4th ICS AAL) at 2nd MCL (right: $p<0.05$, left: $p<0.05$ ). The distance to the closest vital structure in case of a deviation from recommended angle of puncture however was bigger at 4th ICS (ISZ $_{\text {close }}$ right: $p<0.05$, left $p<0.05$ ) (Table 5).

\section{5-year-old children}

Width of the ICS was significantly larger at 2nd ICS (right: $p<0.05$, left: $p<0.05$ ) and mean required depth for successful puncture (CWT) was, with an average difference of 4-5 mm, significantly greater at 2nd ICS compared to 4th ICS (right: $p<0.05$, left: $p<0.05$ ). DVS was significantly larger at 2nd ICS in the left hemithorax $(p<0.05)$ but the difference did not reach statistical significance on the right $(p=0.14) . \mathrm{DVS}_{\text {close }}$ however was larger at 4 th ICS on the right $(p<0.05)$ and roughly the same at 4 th ICS on the left $(p=0.76)$. As a result, the ISZ was greater at 2nd ICS, but the ISZclose was greater at 4th ICS (Table 6).

\section{0-year-old children}

Width of the ICS was significantly greater at 2nd ICS (right: $p<0.05$, left: $p<0.05$ ) and mean required depth for successful puncture (CWT) was significantly greater at 2nd ICS $(2.6 \mathrm{~cm}$ at 2nd ICS and $2.2 \mathrm{~cm}$ at 4th ICS, right: $p<0.05$, left: $p<0.05)$ ). DVS was greater at 2 nd MCL on both sides for correct angle of puncture but did only reach statistical significance on the left side (right: $p=0.13$, left: $p<0.05)$. On the left hemithorax $\mathrm{DVS}_{\text {close }}$ was nearly the same at 2 nd and 4th ICS $(p=0.93)$, and

Table 4 Demographic data

\begin{tabular}{lllll}
\hline Group & Age $($ mean \pm SD $)$ & Male & female & Total \\
\hline 0years & $0.42( \pm 0.32)$ years & $29(=58.0 \%)$ & $21(=42.0 \%)$ & 50 \\
5 years & $5.48( \pm 0.28)$ years & $28(=59.6 \%)$ & $19(=40.4 \%)$ & 47 \\
10years & $10.45( \pm 0.30)$ years & $28(=66.7 \%)$ & $14(=33.3 \%)$ & 42 \\
Total & 5.16 years & $85(=61.2 \%)$ & $54(=38.8 \%)$ & 139 \\
\hline
\end{tabular}


slightly smaller than on the right side $(p=0.11)$. The ISZ was bigger at 2nd ICS (right: $p=0.58$, left $p<0.05$ ), but ISZ $_{\text {close }}$ was bigger at 4th ICS (right: $p<0.05$, left: $p=0.26$ ) (Table 7).

The results for CWT in all age groups are presented graphically in Fig. 2.

\section{Structures directly adjacent to the thoracic wall}

Figure 3 shows the results for "intrapleural safety zone" for the commonly recommended puncture techniques (sagittal puncture at the 2nd ICS MCL and perpendicular puncture at the 4th ICS AAL). As can be seen from the whiskers in the diagram, the safety zone was remarkably small or even zero for some outliers. In the group of infants, the thymus gland was found directly adjacent to the thoracic wall in two infants in the right hemithorax and one infant in the left hemithorax at 2nd ICS MCL. The closest vital structure at this insertion site was the heart, which was found lying only slightly medially to the 2nd ICS MCL on the left. With a puncture directed more medially (at the closest vital structure, $\mathrm{MCL}_{\text {close) }}$ the heart was found adjacent to the chest wall in $8 / 50(16 \%)$ patients on the left and would have been punctured immediately after penetration of the pleura. At 4th ICS AAL, regardless of direction of puncture, no vital structures were found directly adjacent to the chest wall. In one 5-year-old child the heart was found adjacent to the chest wall at 2nd ICS MCL for sagittal puncture on the left. Even a slight deviation (puncture not in the sagittal plane but perpendicular to the chest wall) resulted in the heart being adjacent to the thoracic wall in another child. At 4th ICS AAL no vital structures were found adjacent to the thoracic wall along the path of the needle. In the 10-year-old children, no vital structures were found adjacent to the thoracic wall at 2nd ICS MCL and 4th ICS AAL. Detailed results are shown in the (Additional file 1: Table S1-S3).

\section{Discussion}

Trauma guidelines traditionally recommended 2nd ICS MCL for needle decompression of tension pneumothorax [1,2]. Lately, the 4th and 5th ICS AAL and MAL (midaxillary line) have been recommended as sites of choice by ATLS (Advanced Trauma Life Support) and TCCC (Tactical Combat Casualty Care) guidelines for adult patients $[3,4]$. No specific recommendations are made for children. There are no widely accepted published guidelines on insertion depth in children. Thoracic trauma however, affects around $20 \%$ of moderately to severely injured children [10] and up to $50 \%$ of critically injured children [11]. In an analysis from the German Trauma Registry regarding severe Injuries (AIS $\geq 3$ ), chest trauma was the second most common injury in infants, toddlers and pre-schoolers [12]. Ismail et al. report an incidence of in-hospital need for pleural decompression in paediatric patients with chest trauma of $24.2 \%$ [13]. Tension pneumothorax in children can become rapidly fatal, like in adults. Decompression therefore is often a procedure that has to be performed with high urgency to avoid potentially preventable cardiac arrest. In a cohort of adult traumatic cardiac arrest patients, Kleber et al. report missing or insufficient chest decompression in $37 \%$ of the patients with tension pneumothorax [14]. In a retrospective analysis of children injured during the Afghanistan war, Sokol et al. found that only 14/ 95 patients with an indication for pleural decompression received a prehospital intervention [15]. Especially in children, experience of Emergency Medicine Service

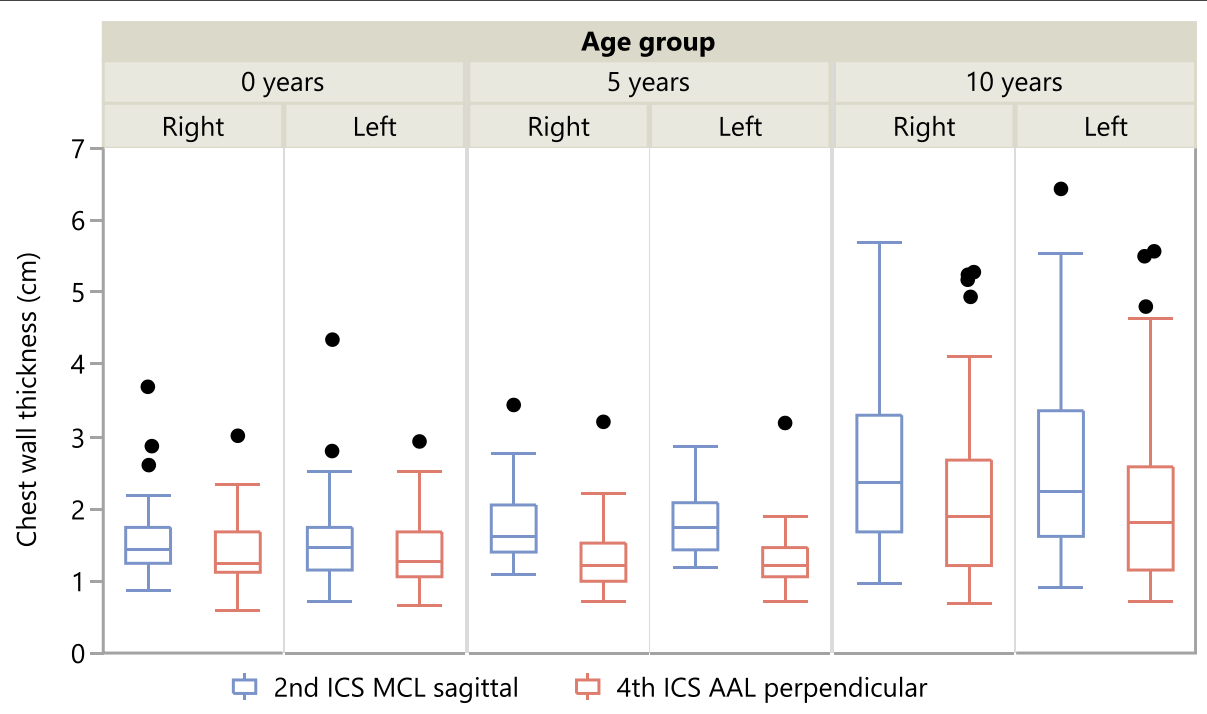

Fig. 2 Chest Wall Thickness. Median, 1st and 3rd Quartile, Minimum, Maximum and Outliers 


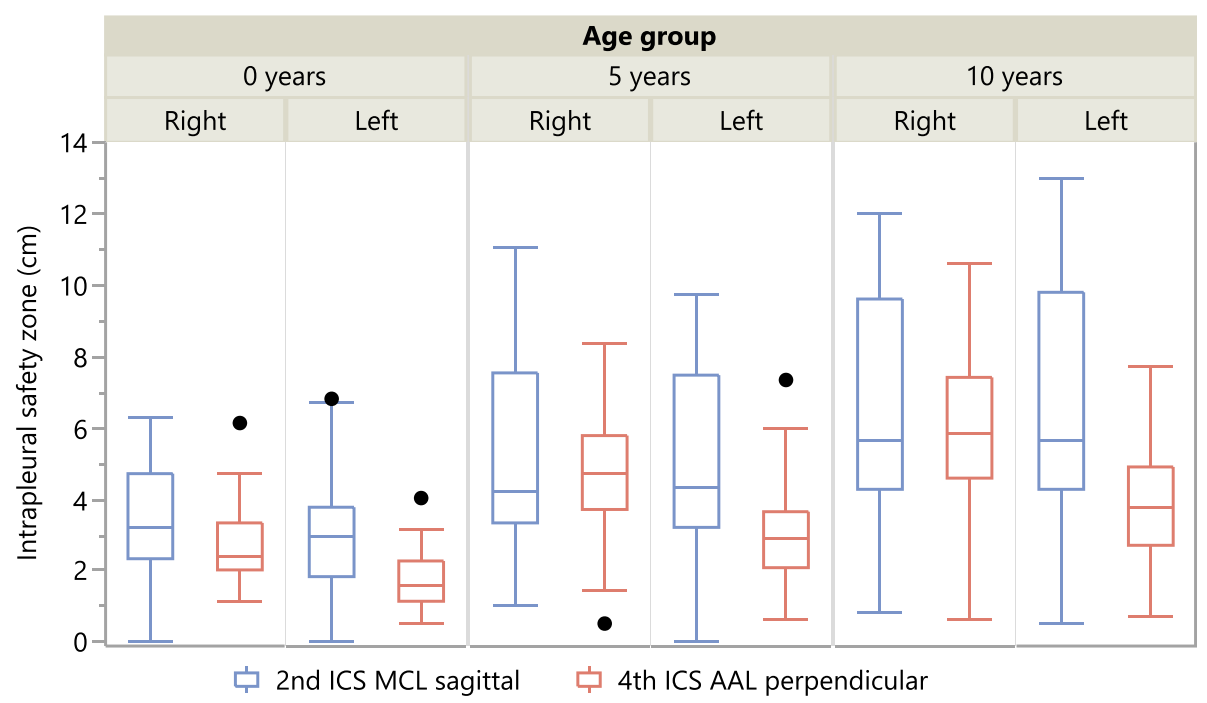

Fig. 3 Intrapleural Safety Zone. Median, 1st and 3rd Quartile, Minimum, Maximum and Outliers

(EMS) personnel performing needle decompression is scarce, let alone open finger thoracostomy. Carlson et al. report an incidence of prehospital pleural decompression in children of only 0.3 per 1000 paediatric EMS responses [16]. This may be due to underdiagnosis, unwillingness to intervene or rarity of the pathology. We therefore conducted this CT-based study to provide guidance regarding the optimal and safest required depth and location of puncture for successful decompression of tension pneumothorax and evaluate the accompanying risks at different puncture sites in three different age groups $(0,5,10$ years).

The width of the intercostal space was significantly lower at 4th ICS AAL in all age groups. The very small width of the ICS in infants (Mean: 4.1-5.8 $\mathrm{mm}$ at the investigated puncture sites, Table 5) poses a significant risk of injuring the intercostal vessels when using large bore cannulas inserted at the incorrect site (intercostal neuromuscular bundle). Laceration of the intercostal artery with subsequent need for surgical intervention as a complication of needle thoracostomy has been described in several case reports for adult patients and has to be taken into account when choosing the cannula bore for decompression [17]. The recommendation of using the cannula with the maximum diameter possible might lead to a serious risk of injury especially in small children [18]. A compromise between better decompression by the higher flow rates of large bore cannulas and the risk of intercostal vessel laceration has to be found. Moreover, the technique of simple open thoracostomy, which is recommended in adults [2] and was recently recommended for children as a result of a Delphi process in the United Kingdom and Ireland [19], is technically not easy for the prehospital or in-hospital provider due to the small intercostal diameters. Decompression failure due to CWT exceeding needle catheter length is a commonly reported phenomenon in adults [6]. In our study mean CWT ranged from $1.56-1.80 \mathrm{~cm}$ in 0 -year-old children, $1.28-1.81 \mathrm{~cm}$ in 5-year-old children and 2.19$2.63 \mathrm{~cm}$ in 10-year-old children. CWT was about 10 $25 \%$ higher at 2nd ICS $\mathrm{MCL}_{\mathrm{sag}}$ compared to 4th ICS $\mathrm{AAL}_{\text {perp }}$. Mandt et al. just recently reported on appropriate needle length for pleural decompression in paediatric patients [20]. The authors measured chest wall thickness in computed tomography scans at 2nd ICS MCL and 4th ICS AAL in four age groups based on Broselow ${ }^{\text {Tw }}$ colour. In the main, the reported results are congruent with the measurements in our study. The CWT reported

Table 5 0-year-old children, all measurements in $\mathrm{cm} \pm \mathrm{SD}$, "**" indicating statistical significance

\begin{tabular}{|c|c|c|c|c|c|c|c|c|c|c|c|c|c|c|}
\hline & \multicolumn{2}{|c|}{ ICS-width } & \multicolumn{2}{|l|}{ CWT } & \multicolumn{2}{|c|}{$\mathrm{CWT}_{\text {close }}$} & \multicolumn{2}{|l|}{ DVS } & \multicolumn{2}{|l|}{ DVS $_{\text {close }}$} & \multicolumn{2}{|l|}{ ISZ } & \multicolumn{2}{|l|}{$I S Z_{\text {close }}$} \\
\hline & right & left & right & left & right & left & right & left & right & left & right & left & right & left \\
\hline \multirow[t]{2}{*}{ 2nd ICS MCL } & 0.55 & 0.58 & 1.52 & 1.56 & 1.43 & 1.40 & 4.96 & 4.48 & 2.44 & 1.96 & 3.44 & 2.92 & 1.01 & 0.56 \\
\hline & {$[ \pm 0.18]$} & {$[ \pm 0.19]$} & {$[ \pm 0.51]$} & {$[ \pm 0.61]$} & {$[ \pm 0.41]$} & {$[ \pm 0.41]$} & {$[ \pm 1.68]$} & {$[ \pm 1.77]$} & {$[ \pm 0.63]$} & {$[ \pm 0.66]$} & {$[ \pm 1.59]$} & {$[ \pm 1.61]$} & {$[ \pm 0.61]$} & {$[ \pm 0.65]$} \\
\hline \multirow[t]{2}{*}{ 4th ICS AAL } & 0.41 & 0.46 & 1.38 & 1.41 & 1.44 & 1.45 & 4.02 & 3.15 & 3.01 & 2.46 & 2.64 & 1.75 & 1.58 & 1.01 \\
\hline & {$[ \pm 0.13]$} & {$[ \pm 0.13]$} & {$[ \pm 0.48]$} & {$[ \pm 0.50]$} & {$[ \pm 0.48]$} & {$[ \pm 0.48]$} & {$[ \pm 1.08]$} & {$[ \pm 0.95]$} & {$[ \pm 0.78]$} & {$[ \pm 0.59]$} & {$[ \pm 0.98]$} & {$[ \pm 0.76]$} & {$[ \pm 0.70]$} & {$[ \pm 0.43]$} \\
\hline$p$ & $<0.05^{*}$ & $<0.05^{*}$ & 0.11 & 0.15 & 0.94 & 0.72 & $<0.05^{*}$ & $<0.05^{*}$ & $<0.05^{*}$ & $<0.05^{*}$ & $<0.05^{*}$ & $<0.05^{*}$ & $<0.05^{*}$ & $<0.05^{*}$ \\
\hline
\end{tabular}


by Mandt el al however is slightly larger than in our study, but the groups used by the authors do not exactly match our age groups, which hinders a direct and exact comparison of the results. Nevertheless, the reported differences are within a range of a few millimetres and do not have to be considered as clinically relevant. Using a $4.5 \mathrm{~cm}$ catheter, decompression would have been successful at all puncture sites in the 0 and 5 -year-old children and in around $90 \%$ of the 10-year-old patients. The use of longer and larger bore catheters however increases the risk of injury to the intercostal vessels and intrathoracic structures. The evaluation of different needle types in the investigated age groups, puncture sites and the most favourable ratio of successful decompression to injury risk is part of another study by our group.

\section{Risk of injury to vital structures}

In presence of a pneumothorax, the air entrapped in the pleural cavity provides a buffer zone for needle puncture, keeping the lung and most likely vital structures away from the chest wall. Clinical diagnosis of a pneumothorax however can be challenging and is likely over-diagnosed especially in the prehospital setting [21]. Thoracic ultrasound is an option to optimize the diagnosis of a pneumothorax but is not universally available prehospital. Nevertheless, false positive diagnosis due to tracheal tube displacement, diaphragmatic rupture, pulmonary contusion or ventilation disturbances is reported in up to $4.5 \%$ [22]. Eckstein et al. report a rate of iatrogenic pneumothorax caused by needle decompression without indication of $2 \%$ [23]. Due to publication bias the true rate is most likely clearly higher. In their aforementioned work, Sokol et al. report of 16 prehospital pleural decompressions in children, of which 2 (12.5\%) were performed without a clear indication [15]. These data show that a false positive diagnosis of tension pneumothorax has to be considered and the risk of injury to intrathoracic vital structures has to be taken into account when choosing the site for needle decompression. So far, to the best of our knowledge, no specific data for children regarding depth to vital intrathoracic organs exist. The risk of injury to vital intrathoracic organs was therefore assessed in further detail in this study.

\section{Risk of injury in infants}

When comparing the two possible puncture sites (2nd ICS $\mathrm{MCL}_{\text {sag }}$ and 4th ICS AAL $\mathrm{Aperp}_{\text {) }}$ ) in infants, CWT was roughly the same and DVS was greater at 2 nd $M C L_{\text {sag }}$. As a result, the ISZ was greatest for 2nd ICS $\mathrm{MCL}_{\text {sag }}$ on both sides of the thorax. The closest vital structure however was found in closer proximity at 2nd ICS MCL. Furthermore, Fig. 3 shows that vital structures (heart, thymus gland) were found directly adjacent to the thoracic wall in several infants. In absence of a pneumothorax the thymus gland would have been hit at the 2nd ICS MCL in two infants on the right and one infant on the left side, even with a correctly directed needle in the sagittal plane. With a misguided puncture, on the left hemithorax, the heart could have been hit immediately after penetration of the thoracic wall in $16 \%$ of the infants. From our point of view, in infants 2nd ICS MCL should only be used after definitive point of care ultrasound/radiographic confirmation of a pneumothorax and the puncture should strictly be performed in the sagittal plane. At 4th ICS AAL no vital structures were found directly adjacent to the chest wall in any direction of puncture in this age group. However, the narrow ICS and the smaller ISZ (DVS - CWT) should be kept in mind at this site of puncture.

\section{Risk of injury to vital structures in 5-year-old children}

CWT was smaller at 4th ICS AAL and DVS was greatest for 2nd ICS $\mathrm{MCL}_{\mathrm{sag}}$. In this age group, the heart was found adjacent to the thoracic wall in one child for sagittal puncture and two children for misguided puncture directed at the closest vital structure at 2nd ICS MCL. Therefore, the risk of puncturing the heart is smaller than in infants, but still present. At 4th ICS AAL no vital structures were directly adjacent, but, as in infants, ISZ (DVS - CWT) was smaller.

\section{Risk of injury to vital structures in 10-year-old children}

For correct direction of puncture (2nd ICS $\mathrm{MCL}_{\mathrm{sag}}$ or 4th ICS $\mathrm{AAL}_{\text {perp }}$ ) no vital structures were directly adjacent to the chest wall. For incorrect angle of needle entry however, the heart could have been injured directly in $16.7 \%$ of the patients at 2nd ICS MCL and $2.4 \%$ at 4th ICS AAL.

Table 6 5-year-old children, all measurements in $\mathrm{cm} \pm$ SD, "**" indicating statistical significance

\begin{tabular}{|c|c|c|c|c|c|c|c|c|c|c|c|c|c|c|}
\hline & \multicolumn{2}{|c|}{ ICS-width } & \multicolumn{2}{|l|}{ CWT } & \multicolumn{2}{|c|}{$\mathrm{CWT}_{\text {close }}$} & \multicolumn{2}{|l|}{ DVS } & \multicolumn{2}{|l|}{ DVS $_{\text {close }}$} & \multicolumn{2}{|l|}{ ISZ } & \multicolumn{2}{|l|}{$I S Z_{\text {close }}$} \\
\hline & right & left & right & left & right & left & right & left & right & left & right & left & right & left \\
\hline \multirow[t]{2}{*}{ 2nd ICS MCL sag } & 1.35 & 1.43 & 1.76 & 1.81 & 1.69 & 1.69 & 6.90 & 6.78 & 3.53 & 3.10 & 5.14 & 4.97 & 1.83 & 1.42 \\
\hline & {$[ \pm 0.31]$} & {$[ \pm 0.36]$} & {$[ \pm 0.48]$} & {$[ \pm 0.48]$} & {$[ \pm 0.46]$} & {$[ \pm 0.48]$} & {$[ \pm 2.43]$} & {$[ \pm 2.67]$} & {$[ \pm 0.70]$} & {$[ \pm 0.91]$} & {$[ \pm 2.46]$} & {$[ \pm 2.68]$} & {$[ \pm 0.77]$} & {$[ \pm 1.03]$} \\
\hline \multirow[t]{2}{*}{ 4th ICS AAL } & 0.72 & 0.83 & 1.34 & 1.28 & 1.42 & 1.37 & 5.98 & 4.29 & 4.01 & 3.00 & 4.64 & 3.00 & 2.58 & 1.63 \\
\hline & {$[ \pm 0.18]$} & {$[ \pm 0.23]$} & {$[ \pm 0.46]$} & {$[ \pm 0.41]$} & {$[ \pm 0.50]$} & {$[ \pm 0.44]$} & {$[ \pm 1.60]$} & {$[ \pm 1.29]$} & {$[ \pm 1.00]$} & {$[ \pm 0.70]$} & {$[ \pm 1.72]$} & {$[ \pm 1.33]$} & {$[ \pm 1.01]$} & {$[ \pm 0.66]$} \\
\hline$p$ & $<0.05^{*}$ & $<0.05^{*}$ & $<0.05^{*}$ & $<0.05^{*}$ & $<0.05^{*}$ & $<0.05^{*}$ & 0.14 & $<0.05^{*}$ & $<0.05^{*}$ & 0.76 & 0.82 & $<0.05^{*}$ & $<0.05^{*}$ & 0.16 \\
\hline
\end{tabular}


Table 7 10-year-old children, all measurements in $\mathrm{cm} \pm \mathrm{SD}$, "*" indicating statistical significance

\begin{tabular}{|c|c|c|c|c|c|c|c|c|c|c|c|c|c|c|}
\hline & \multicolumn{2}{|c|}{ ICS-width } & \multicolumn{2}{|l|}{ CWT } & \multicolumn{2}{|c|}{$\mathrm{CWT}_{\text {close }}$} & \multicolumn{2}{|l|}{ DVS } & \multicolumn{2}{|l|}{ DVS $_{\text {close }}$} & \multicolumn{2}{|l|}{ ISZ } & \multicolumn{2}{|l|}{$\mathbf{I S Z _ { \text { close } }}$} \\
\hline & right & left & right & left & right & left & right & left & right & left & right & left & right & left \\
\hline \multirow[t]{2}{*}{ 2nd ICS MCL $L_{\text {sag }}$} & 1.58 & 1.67 & 2.61 & 2.63 & 2.61 & 2.59 & 9.20 & 9.18 & 4.94 & 4.33 & 6.59 & 6.55 & 2.33 & 1.75 \\
\hline & {$[ \pm 0.31]$} & {$[ \pm 0.34]$} & {$[ \pm 1.15]$} & {$[ \pm 1.23]$} & {$[ \pm 1.17]$} & {$[ \pm 1.28]$} & {$[ \pm 2.94]$} & {$[ \pm 3.36]$} & {$[ \pm 1.17]$} & {$[ \pm 1.43]$} & {$[ \pm 2.98]$} & {$[ \pm 3.29]$} & {$[ \pm 1.08]$} & {$[ \pm 1.29$} \\
\hline \multirow[t]{2}{*}{ 4th ICS AAL } & 1.07 & 1.16 & 2.21 & 2.19 & 2.32 & 2.32 & 8.00 & 6.11 & 5.52 & 4.36 & 5.79 & 3.92 & 3.19 & 2.04 \\
\hline & {$[ \pm 0.33]$} & {$[ \pm 0.37]$} & {$[ \pm 1.33]$} & {$[ \pm 1.30]$} & {$[ \pm 1.34]$} & {$[ \pm 1.39]$} & {$[ \pm 2.29]$} & {$[ \pm 1.81]$} & {$[ \pm 1.76]$} & {$[ \pm 1.44]$} & {$[ \pm 2.12]$} & {$[ \pm 1.53]$} & {$[ \pm 1.36]$} & {$[ \pm 0.9$} \\
\hline$p$ & $<0.05^{*}$ & $<0.05^{*}$ & $<0.05^{*}$ & $<0.05^{*}$ & 0.07 & 0.11 & 0.13 & $<0.05^{*}$ & 0.11 & 0.93 & 0.58 & $<0.05^{*}$ & $<0.05^{*}$ & 0.26 \\
\hline
\end{tabular}

The measures DVS and ISZ suggest a bigger "safety zone" at 2nd ICS in all age groups. Any deviation from correct angle of entry towards the closest vital structure however leads to the opposite result, with $\mathrm{DVS}_{\text {close }}$ and $\mathrm{ISZ}_{\text {close }}$ being greater at 4th ICS. The higher cardiothoracic ratio and, especially in expiration, the more transverse position of the heart in infants, toddlers and pre-school children leads to a closer proximity of the left ventricle to the 2nd ICS MCL on the left hemithorax. This phenomenon is regularly observed in paediatric point-of-care ultrasound examinations of the chest. In summary it seems there is no benefit but increased risk of harm in choosing the 2 nd ICS MCL as insertion site.

\section{Limitations}

This study has several limitations. First of all, measurements were taken in children without pneumothorax. Presence of pneumothorax would minimize the risk of injury to vital structures when puncture is performed under aspiration via syringe and stopped immediately after aspiration of air. However, needle insertion by landmark is not accurate and tension pneumothorax is probably over diagnosed outside the context of POCUS/ radiography. Hence our study findings are relevant. Secondly, the extent of compression of the subcutaneous tissue by the needle tip cannot be measured in CT reliably. Compression might lead to reduced CWT and therefore DVS especially in obese children. Thirdly, we were not able to collect data on the height and weight of the children, which might offer a better correlation with CWT than age [24]. All measurements were recorded by one single investigator. Reproducibility of the measurements was therefore not assessed. Furthermore, we did not record the angle of entry for puncture directed at the closest vital structure. The degree of deviation from the recommended angle that would lead to injury can therefore not be specificied but deviation that would cause injury can be seen. Finally, we identified puncture sites on CT. Ferrie et al. showed a low accuracy among emergency physicians in identifying correct landmarks for needle thoracocentesis in adults with a trend to perform punctures medial to the MCL [25]. In an adult cadaveric study, Inaba et al. found a significantly higher rate of correct needle placement for the 5th ICS compared to the 2nd ICS [26, 27]. In the paediatric population, where bony landmarks are less obvious and smaller spatial relationships are present, there may be increased risk of error in placement position with potential significant iatrogenic injury (e.g. heart) but this was not directly evaluated in our study.

\section{Conclusion}

Based on this study we recommend the 4th ICS AAL as the primary site for needle decompression in tension pneumothorax. As the heart and thymus gland were found directly adjacent to the thoracic wall at 2nd ICS MCL in several children aged 0 and 5 years, this puncture site cannot be recommended unless a pneumothorax in this region is confirmed. The caveat is that although the 4th ICS AAL offers a smaller chest wall thickness, the width of the ICS is narrower and hence the risk of neurovascular bundle injury is slightly increased. However, the difference in width compared to 2nd ICS may not be clinically significant in terms of needle insertion. Deviations from correct angle of entry at 2nd ICS however are accompanied by higher risk of injury than at 4th ICS. To avoid an unnecessarily deep needle penetration, puncture should be performed under guidance by aspiration of air via a syringe and needle movement should be immediately stopped after aspiration of air. Whenever possible ultrasound should be used for confirmation of a pneumothorax, to measure chest wall thickness and confirm lack of underlying vital structure (e.g. heart) before puncture and hence minimize depth of needle insertion and reduce the risk of injuring vital structures. Whenever ultrasound is not available, knowledge on depth to vital structures as well as knowledge concerning chest wall thickness is essential to avoid serious injuries in children. Depth markers on the needle would be helpful for judging depth of needle penetration. Furthermore, a small skin incision prior to puncture can reduce the force needed for advancement of the needle and therefore offer a better control of the depth of puncture. An age appropriate device with a Veress tip could also be an alternative to reduce complications associated with needle decompression. 


\section{Additional file}

Additional file 1: Table S1. Structures directly adjacent to the thoracic wall, 0-year-old children. Table S2. Structures directly adjacent to the thoracic wall, 5-year-old children. Table S3. Structures directly adjacent to the thoracic wall, 10-year-old children. (DOC $67 \mathrm{~kb}$ )

\section{Abbreviations}

AAL: Anterior axillary line; CWT: Chest wall thickness; DVS: Depth to vita

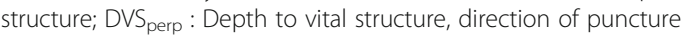
perpendicular to the chest wall; ICS: Intercostal space; MCL: Medioclavicular

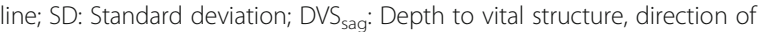

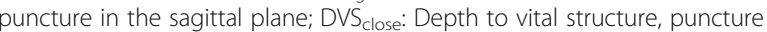
straight in the direction of the closest vital structure; ISZ: Intrapleural safety

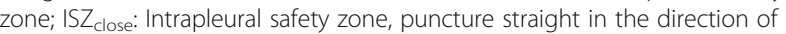
the closest vital; CT: Computed tomography; EMS: Emergency medicine service; ATLS: Advanced trauma life support; TCCC: Tactical combat casualty care

\section{Acknowledgements}

We thank Prof. Brian Burns, Sydney, for critically reviewing this manuscript. We acknowledge financial support by Deutsche Forschungsgemeinschaft within the funding programme Open Access Publishing, by the BadenWürttemberg Ministry of Science, Research and the Arts and by RuprechtKarls-Universität Heidelberg.

\section{Funding}

Financial support (50\% of the Article Processing Charge) was received by Deutsche Forschungsgemeinschaft.

\section{Availability of data and materials}

All data generated or analysed during this study are included in this published article and its supplementary files.

\section{Authors' contributions}

TT contributed by planning the study, analyzing and interpreting the data and was the major contributor in writing the manuscript. GL collected and analyzed the data. LW, TV, MR, MS and MW were involved in planning the study and analyzing and interpreting the data. $\mathrm{HH}$ contributed by planning the study, analyzing and interpreting the data and was a major contributor in writing the manuscript. All authors read and approved the final manuscript.

\section{Authors' information}

All authors work at Mannheim University Medical Center. TT, TV, MR and MS work as consultants in anesthesiology and prehospital emergency medicine, with a strong focus on paediatric anesthesiology and paediatric emergency medicine. GL is writing his doctoral thesis on decompression of tension pneumothorax in children. LW is head of the Department of Paediatric Surgery and a well-known expert in paediatric traumatology and paediatric thoracic surgery. MW and $\mathrm{HH}$ are radiology consultants with a focus on cardiothoracic imaging and paediatric radiology.

\section{Ethics approval and consent to participate}

Ethics approval for this study was obtained from Medical Ethics Committee II, Medical Faculty Mannheim, Mannheim University (Reference number: 2013-818R-MA)

\section{Competing interests}

The authors declare that they have no competing interests.

\section{Publisher's Note}

Springer Nature remains neutral with regard to jurisdictional claims in published maps and institutional affiliations.

\section{Author details}

'Department of Anaesthesiology and Intensive Care Medicine, University Medical Center Mannheim, Theodor-Kutzer-Ufer 1-3, 68167 Mannheim, Germany. ${ }^{2}$ Department of Paediatric Surgery, Mannheim University Medical
Center, Theodor-Kutzer-Ufer 1-3, 68167 Mannheim, Germany. ${ }^{3}$ DRF Stiftung Luftrettung gemeinnützige $A G$, Filderstadt, Germany. ${ }^{4}$ Institute of Clinical Radiology and Nuclear Medicine, University Medical Center Mannheim, Theodor-Kutzer-Ufer 1-3, 68167 Mannheim, Germany.

Received: 3 February 2019 Accepted: 26 March 2019

Published online: 16 April 2019

\section{References}

1. American College of Surgeons. Advanced trauma life support ${ }^{\oplus}, 9^{\text {th }}$ ed. Chicago: American College of Surgeons; 2013.

2. Bouillon B, Begleitung M, Pieper D et al. S3 - Leitlinie Polytrauma / Schwerverletzten-Behandlung. AWMF Register-Nr. 012/019. 2016. https:// www.awmf.org/uploads/tx_szleitlinien/012-019|_S3_Polytrauma_ Schwerverletzten-Behandlung_2017-08.pdf.

3. Montgomery HR, Butler FK, Giebner SD et al. TACTICAL COMBAT CASUALTY CARE (TCCC / TC3) https://rmf.ims.allogy.com/pf.tlx/Z03ZHMZHr5TL. Accessed 20 Nov 2018.

4. American College of Surgeons. Advanced Trauma Life Support ${ }^{\oplus}$, 10th ed. Chicago: American College of Surgeons; 2018.

5. Advanced Life Support Group. Advanced Pediatric Life Support, $6^{\text {th }}$ Edition. Hoboken: Wiley-Blackwell; 2016.

6. Laan D, Vu T, Thiels $C$, et al. Chest wall thickness and decompression failure: a systematic review and meta-analysis comparing anatomic locations in needle thoracostomy. Injury. 2016;47:797-804.

7. Hecker M, Hegenscheid $K$, Völzke $H$, et al. Needle decompression of tension pneumothorax: population-based epidemiologic approach to adequate needle length in healthy volunteers in Northeast Germany. J Trauma Acute Care Surg. 2016;80:119-24

8. Aho J, Thiels C, El Khatib M, et al. Needle Thoracostomy: clinical effectiveness is improved using a longer Angiocatheter. J Trauma Acute Care Surg. 2016;80:272-7.

9. Chang S, Ross S, Kiefer D, et al. Evaluation of 8.0-cm needle at the fourth anterior axillary line for needle chest decompression of tension pneumothorax. J Trauma Acute Care Surg. 2014;76:1029-34.

10. Naqvi G, Johansson G, Yip G, Rehm A, Carrothers A, Stöhr K. Mechanisms, patterns and outcomes of paediatric polytrauma in a UK major trauma Centre. Ann R Coll Surg Engl. 2017;99:39-45.

11. Gatzka C, Begemann P, Wolff A, Zörb J, Rueger J, Windolf J. Verletzungsmuster und klinischer Verlauf polytraumatisierter Kinder im Vergleich mit Erwachsenen: Eine 11-Jahres-Analyse am Klinikum der Maximalversorgung. Unfallchirurg. 2005;108:470-80.

12. Wyen $\mathrm{H}$, Jakob $\mathrm{H}$, Wutzler $\mathrm{S}$, et al. Prehospital and early clinical care of infants, children and teenagers compared to an adult cohort: analysis of 2961 children in comparison to21435 adult patients from the trauma registry of DGU in a 15-year period. Eur J Trauma Emerg Surg. 2010;36:300-7.

13. Ismail MF, Al-Refaie RI. Chest trauma in children, single center experience. Arch Bronconeumol. 2012:48:362-6.

14. Kleber C, Giesecke M, Lindner T, Haas N, Buschmann C. Requirement for a structured algorithm in cardiac arrest following major trauma: epidemiology, management errors, and preventability of traumatic deaths in Berlin. Resuscitation. 2014:85:405-10.

15. Sokol K, Black G, Azarow K, Long W, Martin W, Eckert M. Prehospital interventions in severely injured pediatric patients: rethinking the $A B C s$. J Trauma Acute Care Surg. 2015;79:983-9.

16. Carlson J, Gannon E, Clay Mann N, et al. Pediatric out-of-hospital critical procedures in the United States. Pediatr Crit Care Med. 2015;16:e260-7.

17. Yacovone ML, Kartan R, Bautista M. Intercostal artery laceration following thoracentesis. Respir Care. 2010;55:1495-8.

18. Heinrich M. Kinderchirurgie: Basiswissen und Praxis. Germering: W. Zuckschwerdt Verlag; 2012

19. Vassallo J, Nutbeam T, Rickard AC, et al. Paediatric traumatic cardiac arrest: the development of an algorithm to guide recognition, management and decisions to terminate resuscitation. Emerg Med J. 2018;35:669-74.

20. Mandt MJ, Hayes K, Severyn F, Adelgais K. Appropriate needle length for emergent pediatric needle Thoracostomy utilizing computed tomography. Prehosp Emerg Care. 2019:9:1-9. https://doi.org/10.1080/10903127.2019. 1566422 [Epub ahead of print].

21. Waydhas C, Sauerland S. Pre-hospital pleural decompression and chest tube placement after blunt trauma: a systematic review. Resuscitation. 2007;72:11-25. 
22. Lechleuthner A, Bouillon B, Neugebauer, Mennigen R, Thiling T. Prehospital chest tubes - incidence and analysis of iatrogenic injuries in the emergency medical service Cologne. Theor Surg. 1994;9(4):220-6.

23. Eckstein M, Suyehara D. Needle thoracostomy in the prehospital setting. Prehospital Emerg Care. 1998;2:132-5.

24. Powers WF, Clancy TV, Adams A, West T, Kotwall C, Hope W. Proper catheter selection for needle thoracostomy: a height and weight-based criteria. Injury. 2014;45:107-11.

25. Ferrie EP, Collum N, McGovern S. The right place in the right space? Awareness of site for neddle thoracocentesis. Emerg Med J. 2005;22:788-9.

26. Inaba K, Karamanos E, Skiada D, et al. Cadaveric comparison of the optimal site for needle decompression of tension pneumothorax by prehospital care providers. J Trauma Acute Care Surg. 2015;79:1044-8.

27. Inaba K, Branco BC, Eckstein M, et al. Optimal positioning for emergent needle thoracostomy: a cadaver-based study. J Trauma. 2011;71:1099-103.

Ready to submit your research? Choose BMC and benefit from:

- fast, convenient online submission

- thorough peer review by experienced researchers in your field

- rapid publication on acceptance

- support for research data, including large and complex data types

- gold Open Access which fosters wider collaboration and increased citations

- maximum visibility for your research: over $100 \mathrm{M}$ website views per year

At $\mathrm{BMC}$, research is always in progress.

Learn more biomedcentral.com/submissions 